\title{
The significance of feedback control for chemical sensors
}

\author{
P. Bergveld \\ MESA Research Institute, Faculty of Electrical Engineering, University of Twente, P.O. Box 217, 7500 AE Enschede (Netherlands)
}

(Received May 7, 1991; accepted October 2, 1991)

\begin{abstract}
The conventional way of applying chemical sensors is in an open-loop configuration. A parameter of the chemical domain, such as a gas or ion concentration, is converted into a parameter of the mechanical or electrical domain, often with non-linear transfer characteristics. The paramagnetic oxygen sensor and the ISFET-based urea sensor are treated as typical examples. Such an open-loop procedure is inconceivable in modern electronic and control systems, where the application of feedback is common practice. The question may arise why this principle is hardly adopted in the development of chemical sensors, to stabilize the signal conversion and to linearize the sensor functioning. Based on the analysis of a transistor $V-I$ converter in open-loop and closed-loop configurations, the successful performance of feedback in the case of the paramagnetic oxygen sensor and the ISFET-based urea sensor is analysed. That most other chemical sensors are still used in an open-loop configuration is obviously due to the lack of chemical actuators that can be integrated with the corresponding chemical sensors. It is therefore stated that it will be important to pay more attention to the development of chemical actuators in order to make progress in the field of chemical sensors.
\end{abstract}

\section{Introduction}

Electrochemical sensors, both conventional as well as modern types that are at present being developed on the basis of chip technology, can be roughly divided into potentiometric and amperometric sensors. In a potentiometric sensor a chemical quantity is converted into an electrical potential, whereas in an amperometric sensor it is converted into an electrical current. The resulting electrical potential or current is directly related to the chemical quantity to be measured, for instance, the concentration of ions or the partial pressure of a gas. Examples of potentiometric sensors are the many ion-selective sensors based on the existence of a surface or membrane potential as a function of the concentration of a specific ion. An example of an amperometric sensor is the oxygen sensor, based on the reduction of dissolved oxygen at a cathode of an electrolytic cell. It is essential to note that the sensors measure an equilibrium state and are therefore applied in a static way.

Based on these direct sensors, a variety of indirect sensors has been developed in which an intermediate layer provides the conversion of a certain substrate into a related concentration of ions or partial gas pressure. Many enzyme sensors are based on the immobilization of a layer of specific enzymes on top of a potentiometric or amperometric sensor. Due to an enzymatic reaction in this layer, a substrate is converted locally into species to which the underlying sensor is sensitive.

For the application of the direct as well as the indirect sensors, the characteristic behaviour of the signal conversion from the chemical to the electrical domain is of course roughly known. However, this behaviour may alter as a function of time as a consequence of the nature of the (bio)chemical reactions on which these types of sensors rely. For instance, an ion-selective membrane electrode provides, within a certain range, a potential as a linear function of pI, but its sensitivity as well as its baseline will change with time as a function of the composition of the liquid in which it is immersed. Also fouling of the membrane may drastically degrade the proper functioning of the electrode. Most enzyme electrodes respond in a very non-linear way due to the strong dependence of enzyme activity on its environment. Also, competition 
with other occurring chemical reactions will strongly influence the signal conversion.

The application of sensors in general is traditionally open-loop, with some exceptions for mechanical and magnetic sensors. The static direct and indirect electrochemical sensors as mentioned before are also used in an open-loop configuration, which leads to the necessity of frequent calibration. The repetition rate of the calibration depends mainly on the required accuracy of a measurement and the experience with the particular sensor. In addition, information and algorithms may be necessary for the final determination of the chemical quantity to be monitored.

The problem will be analysed in more detail below and two examples, one for the measurement of oxygen and one for the measurement of urea, will be treated. This will be done according to the analysis of the well-known example of a transistor amplifier, for which the stable sensitivity and linearity are the result of applying the feedback principle.

\section{The voltage-to-current $(V-I)$ converter}

Although the signal conversion is from the electrical domain to the electrical domain, a transistor can nevertheless be compared with a chemical sensor because it also converts an input signal, in this case a voltage, into an output signal, in this case a current, via a far-from-ideal characteristic. As a $V-I$ converter, schematically shown in Fig. 1(a), the single transistor converts the input voltage $V_{\text {in }}=V_{\text {be }}$ into the output current $I_{\mathrm{c}}$ according to

$I_{\mathrm{c}}=I_{\mathrm{s}} \exp \left(V_{\mathrm{be}} / V_{T}\right)$

where $I_{\mathrm{s}}$ is a constant used to describe the transfer characteristic of the transistor in the forwardactive region, depending on many solid-state parameters, and $V_{T}=k T / q$.

Because of the non-linearity, transistors are never used in an open-loop configuration as shown in Fig. 1(a), but in a closed-loop system as given in Fig. 1(b). Here a comparator/amplifier is added, which regulates a feedback current $I_{\mathrm{f}}$ through a resistor $R$, resulting in a $V / I$ conversion according to

$I_{\mathrm{f}}=1 / R\left[V_{\text {in }}-V_{\mathrm{be}}+V_{\mathrm{ref}} R / R_{\mathrm{c}}\right]$

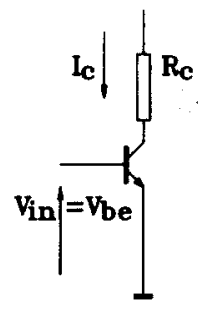

(a)

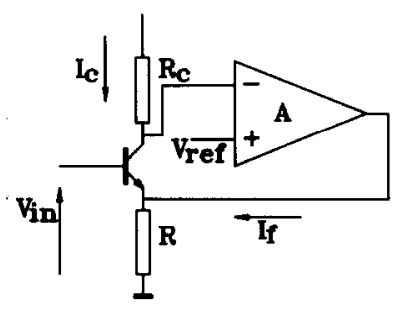

(b)

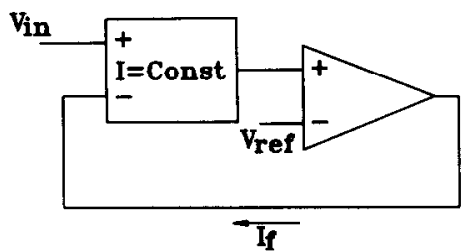

(c)

Fig. 1. (a) Single transistor $V / I$ converter, open-loop. (b) Transistor $V / I$ converter, closed-loop. (c) Block diagram of (b).

where $V_{\text {ref }}$ is a set point, which may bias the system to $I_{\mathrm{f}}=0$ for $V_{\text {in }}=0$. The basic principle is schematically represented in Fig. 1(c).

This simple example clearly shows the essential design rules for a feedback system, for which it will be worthwhile to consider the application with respect to chemical sensors. The most essential property of the system is that the feedback signal is of the nature of the converted signal, in the transistor case a current, which is kept at a constant level, provided that the loop gain is high enough and the phase rules are also obeyed. The resulting transfer characteristic is highly linearized, because the bias point of the transistor (transducer) is stable. Note that $I_{\mathrm{f}}$ is still a function of $V_{\text {be }}$, which means, according to eqn. (1), that the dependence of solid-state parameters is not cancelled. Because these parameters are not influenced by environmental conditions, except for the temperature, this is not dramatic for the $V / I$ conver- 
sion, but a similar dependence in the case of chemical sensors may be worse, regardless of the application of feedback.

\section{The paramagnetic oxygen sensor}

Due to the unpaired electron-spin configuration of the oxygen molecule, the magnetic moment is not neutralized, resulting in a strong magnetic property of oxygen in comparison to many other gases. This means that oxygen can be selectively distinguished from other gases. The strength of the interaction between a molecule and a magnetic field is called magnetic susceptibility. In the case of oxygen, the magnetic susceptibility is positive, which phenomenon is called paramagnetism. Nitrogen, on the other hand, has a weak negative susceptibility, which phenomenon is called diamagnetism. The force $F$ on a para- or diamagnetic molecule in a magnetic field is proportional to the product of its susceptibility $\chi$, the strength of the magnetic field $H$ and the magnetic field gradient $\mathrm{d} H / \mathrm{d} x$ :

$F=\chi H \mathrm{~d} H / \mathrm{d} x$

Magnetic field gradients are present between the wedge-shaped poles of a permanent magnet. Oxygen molecules in such a field will therefore experience a force in the direction of the highest magnetic field, according to eqn. (3). Nitrogen molecules will experience a force in the opposite direction. Pauling et al. [1] made use of this phenomenon by constructing a mechanical system which is able to convert these forces into a measurable signal. They mounted between the wedgeshaped poles of a permanent magnet a quartz fibre to which a small mirror was attached, together with a glass rod, having at the ends sealed glass dumb-bells containing nitrogen gas. The set-up is schematically drawn in Fig. 2(a).

In the gradient of the magnetic field, oxygen molecules surrounding the glass spheres will in fact replace the nitrogen molecules due to the opposite forces and vice versa. This resulting differential force causes a torsion of the twin dumb-bell construction, proportional to the partial pressure of oxygen in the surrounding gas. The torsion is measured by means of the measurement of the deflection of a light beam, reflected by the mirror.

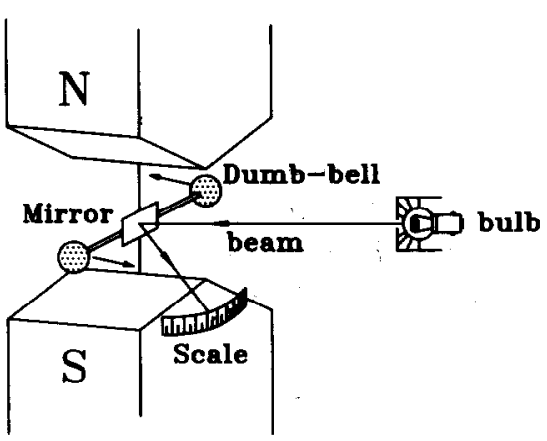

(a)

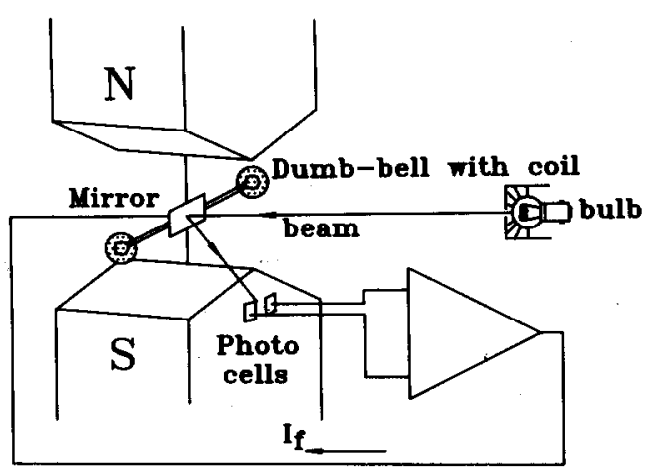

(b)

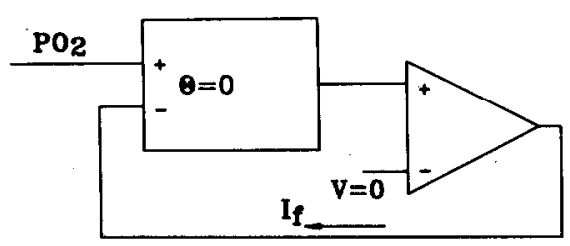

(c)

Fig. 2. (a) Schematic representation of paramagnetic oxygen sensor in open-loop configuration. (b) Paramagnetic oxygen sensor with feedback. (c) Block diagram of (b).

Assuming that the overall relation between the partial pressure of oxygen, $P_{\mathrm{O}_{2}}$, and the angle of rotation $\theta$ is $\alpha$, the measurement of $P_{\mathrm{O}_{2}}$ can be described as

$\theta=\alpha H(\mathrm{~d} H / \mathrm{d} x) P_{\mathrm{O}_{2}}$

This relation is very non-linear because of $\mathrm{d} H / \mathrm{d} x$, comparable with the open-loop transistor application as given in Fig. 1(a). Therefore the original Pauling instrument has been modified with a feedback loop according to the recipe described in the previous Section. Because $\theta$ is the output signal of the conversion from the chemical to the mechanical domain, the feedback should result in an action which keeps the rotation angle zero. This is 
provided by the addition of coils which are integrated with the dumb-bells. The current through the coils is controlled by the output of a differential amplifier whose inputs are connected to two photocells, which detect the light-beam deflection.

The final result is that the system now operates with a fixed bias point at $\theta=0$, with a feedback current $I_{\mathrm{f}}$ through the coils that is a linear function of the partial oxygen pressure of the surrounding gas:

$I_{\mathrm{f}}=\beta H P_{\mathrm{O}_{2}}$

The system is represented by Fig. 2(b) and, for comparison with Fig. 1(c), is schematically drawn in Fig. 2(c).

As in the example of the transistor, the $I_{\mathrm{f}} / P_{\mathrm{O}_{2}}$ relation is still a function of some physical parameters (see eqn. (5)), of which $\beta$ especially is very sensitive to vibrations. Nevertheless, Servomex Ltd succeeded in constructing a good laboratory instrument for clinical application [2].

\section{The FET-based urea sensor}

Layers of immobilized enzymes are often used to convert a chemical signal, for which no direct sensor is available, into a chemical signal for which a sensor exists. A well-known example is the measurement of urea by means of the enzyme urease, resulting in a $\mathrm{pH}$ change in the immobilized enzyme layer:

urea $+3 \mathrm{H}_{2} \mathrm{O} \stackrel{\text { urease }}{,} 2 \mathrm{NH}_{4}^{+}+\mathrm{HCO}_{3}^{-}+\mathrm{OH}^{-}$

The urease is usually immobilized in a membrane that covers a pH sensor. The conventional bulbshaped $\mathrm{pH}$ glass membrane is less suitable for deposition of a membrane, but the pH-ISFET is a much better candidate, due to its planar construction, small dimensions and fast response. The first FET-based urea sensor was published by Miyahara et al. in 1983 [3]. They described a urea and an acetylcholine ENFET, measured with respect to a reference FET (REFET) on which glycine was immobilized instead of urease and acetylcholinesterase, respectively, for the ENFETs. The differential output signal of the ENFET/REFET system as represented by Fig. 3(a) is given by

$V_{\text {out }}=E(\alpha / \beta)[\mathrm{S}]$

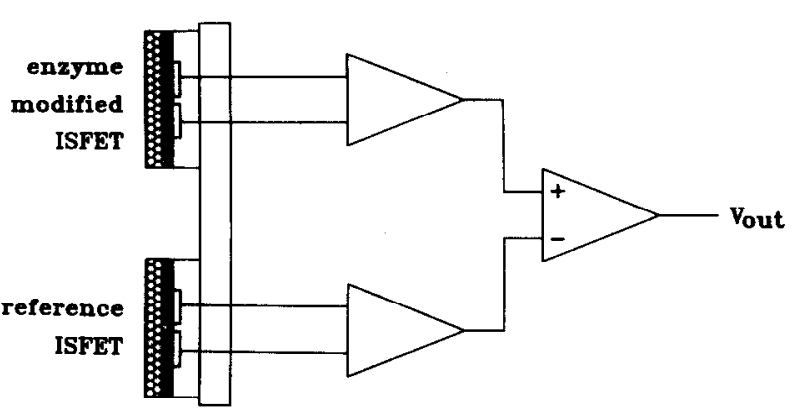

(a)

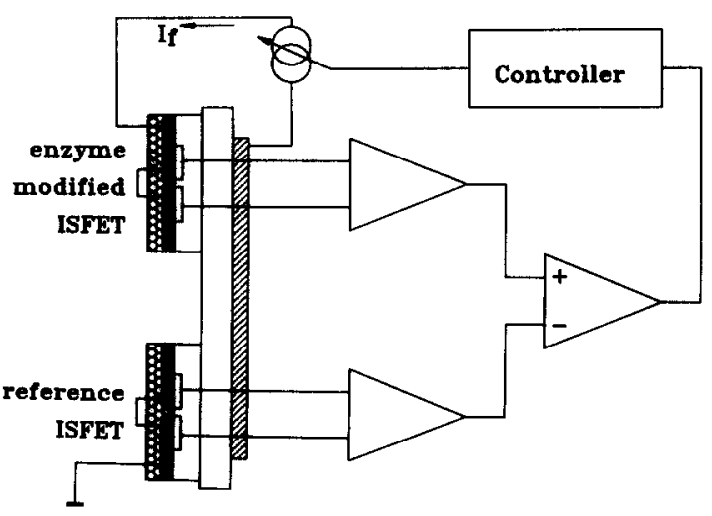

(b)

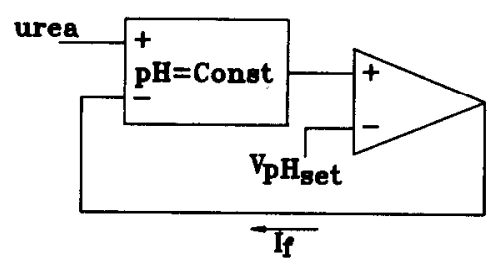

(c)

Fig. 3. (a) Open-loop configuration of ENFET/REFET measurement set-up. (b) ENFET/REFET system with coulometric fccdback. (c) Block diagram of (b).

where [S] is the urea concentration, $\alpha$ is the sensitivity of the ISFET in $\mathrm{mV} / \mathrm{pH}, \beta$ is the buffer capacity of the sample in $\mathrm{mol} / \mathrm{l} / \mathrm{pH}$ and $E$ is the enzymatic sensitivity $\left(\left[\mathrm{OH}^{-}\right] /[\mathrm{S}]\right)$.

The last parameter represents the change in equivalents of $\mathrm{OH}^{-}$ions in the membrane as a function of the substrate concentration [S] per unit of time. $E$ is determined by the enzyme load of the membrane, the enzyme kinetics, the diffusion constants of the relevant species in the membrane and the $\mathrm{pH}$ inside the membrane. The last dependency means that with a urea concentration of, for example, $2 \mathrm{mmol}$ in a sample of $\mathrm{pH}=7$, the membrane $\mathrm{pH}$ may change from 7 to approximately 9 , which 
decreases $E$ to about $30 \%$ of its original value. This means that the $V_{\text {out }} /[\mathrm{S}]$ curves are strongly s-shaped. Moreover, the position of the curve along the [S] axis depends strongly on the buffer capacity $\beta$, according to eqn. (7). This buffer dependency as well as the non-linearity makes it desirable to investigate the possibility of feedback for improving the sensor characteristics.

In comparison with the systems described in the previous Sections and illustrated in Figs. 1(c) and 2(c), respectively, it is necessary for the urea sensor that the $\mathrm{pH}$ changes induced in the membrane by the conversion of urea are compensated by feedback. Fortunately ISFETs can be provided relatively easily with a $\mathrm{pH}$ actuator, consisting of a noble metal film closely surrounding the gate area [4].

A current through this electrode with respect to a remote counter electrode will produce protons or hydroxyl ions, depending on the current direction, due to the electrolysis of water, known as coulometry. The ENFET shown in Fig. 3(a) has therefore to be provided with an actuator electrode before the urea-containing membrane is deposited. A control system has been designed in such a way that the $\mathrm{pH}$, as measured by the ISFET, is kept at the value of the sample solution, as measured by the REFET [5]. This system is schematically given in Fig. 3(b).

For small changes in $\mathrm{pH}$, the buffer capacity may be considered as constant and the coulometric actuator will operate in a linear way:

$V_{\text {out }}=A(\alpha / \beta) I$

where $A(\mathrm{~mol} / \mathrm{l})$ is the sensitivity parameter of the sensor/actuator system, determined by the actuator dimensions and the diffusion of protons and hydroxyl ions in the membrane. Because both of these can be considered as constants, $A$ will also be constant.

The control system delivers a coulometric current which continuously compensates the $\mathrm{pH}$ changes produced by the enzymatic reaction. Hence in the equilibrium state $V_{\text {out.current }}=-V_{\text {out,enzyme }}$. Thus a combination of eqns. (7) and (8) results in

$I=-E / A[\mathrm{~S}]$

Equation (9) shows that the output current of the feedback system as a function of the urea concentration is thus independent of the buffer capacity. Moreover, the output of the system is strongly linearized, because the membrane $\mathrm{pH}$ is kept constant, resulting in a constant parameter $E$.

In Fig. 3(b) the pH in the membrane is kept at the $\mathrm{pH}$ value of the sample solution, due to comparison with the REFET, which contains a membrane similar to that of the ENFET, but without the addition of urease.

If for a proper functioning of the enzyme a more optimal $\mathrm{pH}$ in the membrane is necessary, the membrane can also be kept continuously at this set $\mathrm{pH}$ level [6]. The block diagram of this system is given in Fig. 3(c), for comparison with Figs. 1(c) and 2(c).

Note that the $I /[\mathrm{S}]$ conversion is still a function of the enzyme activity $E$, which means that feedback does not prevent loss of sensitivity if the enzyme load decreases due to release of the enzyme or as a result of degradation of the biological material.

\section{Conclusions}

Summarizing the three examples of feedback systems described here, it is obvious that linearization is always achieved because the operational bias point of the signal converter is fixed to a preset value. In all three cases the output signal is a feedback current that is related to the input signal by means of physical constants in the case of the $V-I$ converter and the $P_{\mathrm{O}_{2}}$ sensor, but by chemical constants in the case of the urea sensor. Here an important difference shows up. Physical constants, like donor and intrinsic carrier concentrations ( $V_{-} I$ converter) and a magnetic field strength $\left(P_{\mathrm{O}_{2}}\right.$ sensor) are hardly influenced by environmental conditions, but chemical constants, like enyzme activity (urea sensor), usually change as a function of time. Application of feedback does not circumvent this problem.

Another difference between the three systems is that the open-loop characteristics of the converters are in fact replaced by the conversion characteristic of the feedback converter. In the case of the $V-I$ converter this is a simple resistor; for the $P_{\mathrm{O}_{2}}$ sensor it is a magnetic field and in the case of the urea sensor it is the coulometric actuator. Here 
again it has to be mentioned that the chemical actuator is less stable than its physical counterparts due to the nature of chemical reactions, which may be influenced by fouling or side reactions, for instance. Nevertheless, besides linearization, specific interferences may be cancelled by the feedback, as occurs with the buffer dependency in the case of the urea sensor.

As a final conclusion, it may be stated that the application of feedback for chemical sensors is very useful, but the systems still have to be calibrated more often than their physical counterparts. That chemical sensors with internal feedback hardly exist is mainly due to a lack of chemical actuators that can be integrated with the corresponding sensors. Therefore, it is advisable to stimulate the development of chemical actuators.

\section{Acknowledgements}

The author greatly appreciates the helpful discussions with Ir E. L. de Weerd and Dr Ir W. Olthuis, and would like to thank G. Bultstra for the design and production of the Figures.

\section{References}

1 L. Pauling, R. Wood and J. H. Sturdivant, An instrument for determining the partial pressure of oxygen in a gas, J. Am. Chem. Soc., 63 (1946) 795.

2 F. R. Ellis and J. F. Nunn, The measurement of gaseous oxygen tension utilizing paramagnetism: an evaluation of the Servomex OA150 analyzer, Brit. J. Anaesth. 40 (1968) 569-578.

3 Y. Miyahara, F. Matsu, T. Moriizumi, H. Matsuoka, I. Karube and S. Suzuki, Micro enzyme sensor using semiconductor and enzyme-immobilization techniques, Proc. Int. Meeting on Chemical Sensors, Fukuoka, Japan, Sept. 19-22, 1983, Analytical Chemistry Symposia Series, Vol. 17, Elsevier, Amsterdam, 1983, pp. 501-506.

4 B. H. van der Schoot and P. Bergveld, An ISFET-based microlitre titrator: integration of a chemical sensor-actuator system, Sensors and Actuators, 8 (1985) 11-22.

5 B. H. van der Schoot and P. Bergveld, ISFET based enzyme sensors, Biessensurs, 3 (1988) 161-186.

6 B. H. van der Schoot and P. Bergveld, Evaluation of the sensor properties of the pH-static enzyme sensor, Anal. Chim. Acta., 233 (1990) 49-57. 